November 2000

\title{
AdS/CFT correspondence, conformal anomaly and quantum corrected entropy bounds
}

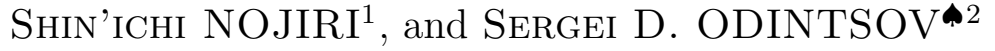 \\ Department of Applied Physics \\ National Defence Academy, Hashirimizu Yokosuka 239-8686, JAPAN \\ \$ Instituto de Fisica de la Universidad de Guanajuato, Lomas del Bosque \\ 103, Apdo. Postal E-143, 37150 Leon,Gto., MEXICO
}

\begin{abstract}
The role of conformal anomaly in AdS/CFT and related issues is clarified. The comparison of holographic and QFT conformal anomalies (with account of brane quantum gravity contribution) indicates on the possibility for brane quantum gravity to occur within AdS/CFT set-up. 3d quantum induced inflationary (or hyperbolic) brane-world is shown to be realized in frames of AdS3/CFT2 correspondence where the role of $2 \mathrm{~d}$ brane cosmological constant is played by effective tension due to two-dimensional conformal anomaly. The dynamical equations to describe 4d FRW-Universe with account of quantum effects produced by conformal anomaly are obtained. The quantum corrected energy, pressure and entropy are found. Dynamical evolution of entropy bounds in inflationary Universe is estimated and its comparison with quantum corrected entropy is done. It is demonstrated that entropy bounds for quantum corrected entropy are getting the approximate ones and occur for some limited periods of evolution of inflationary Universe.
\end{abstract}

\footnotetext{
${ }^{1}$ nojiri@cc.nda.ac.jp

${ }^{2}$ On leave from Tomsk State Pedagogical University, 634041 Tomsk, RUSSIA. odintsov@ifug5.ugto.mx, odintsov@mail.tomsknet.ru
} 


\section{Introduction}

AdS/CFT correspondence [1] in its simplest version shows remarkable duality between classical higher dimensional gravity and (brane) QFT living in less dimensions. The power of dualities is explicitly expressed in this new principle. It demonstrates the universality of high energy physics in description of areas which seem to be quite distant. As it happens usually, providing new ways in the resolution of well-known problems AdS/CFT puts also new questions. Just to mention some of them: The description of brane quantum gravity in terms of AdS/CFT set-up? The consistent embedding of Randall-Sundrum orbifold compactification as warped compactification in string theory? AdS/CFT basis for (quantum) entropy bounds origin? It is expected that holographic conformal anomaly [2] and its counterpart, quantum field conformal anomaly (for a review, see [3]) should play an essential role in the study of above as well as related problems.

In the present paper we discuss the role played by conformal anomaly in various aspects of AdS/CFT and in related issues. First of all, in the next section the comparison of holographic and QFT conformal anomalies is done. The account of quantum gravity (brane gravity) contribution in quantum conformal anomaly is made. As a result, it follows that holographic conformal anomaly may be equal to quantum conformal anomaly not only for super Yang-Mills theory but also for (non)supersymmetric matter theory with brane gravity. This indicates that brane (Einstein or Weyl) gravity has some chances to occur in AdS/CFT set-up, at least as next-to-leading order effect.

As the other application of conformal anomaly the scenario of refs. [4, 5] is extended to d3 brane-worlds. According to this scenario, originally suggested for d5 brane-worlds, Randall-Sundrum Universe is realized in AdS3/CFT2 correspondence as warped compactification (kind of holographic RG flow). The essential role is played by $2 \mathrm{~d}$ conformal anomaly (brane cosmological constant is fixed) which introduces the effective brane tension. As a result quantum induced inflationary or hyperbolic brane-world Universe occurs. The details of such construction are described in third section.

In fourth section, again using conformal anomaly (or anomaly induced effective action) the dynamical evolution equations describing 4d FRW-

Universe are defined. The same equations are obtained by two different formalisms where the account of quantum effects in energy/pressure is done. 
It is mentioned that these equations which admit de Sitter solution are the basis for anomaly driven inflation which is considered to be quite realistic nowdays. (4d anomaly driven inflation [6] is closely related with anomaly driven inflationary brane-worlds [4, 5, 7] as it is noted in fourth section). The quantum corrected energy and pressure are defined. It is remarkable that quantum energy and pressure may include the contributions from quantum gravity itself (or in another way, Einstein gravity is modified by anomaly induced effective action which accounts quantum effects of matter and gravity via the corresponding coefficients of conformal anomaly). Following to recent proposal by E. Verlinde 8 on the study of dynamical evolution of entropy bounds the attempt to introduce quantum entropy is done. Considering de Sitter space as an example it is suggested that large quantum contribution to entropy may destroy well-accepted entropy bounds (say of BekensteinHawking type). As a result the entropy bounds become approximate ones and occur only for some time-limited periods of Universe evolution. They also evolve and with the Universe expansion they may appear in different way as we speculate. Brief summary and some outlook is presented in last section.

\section{AdS/CFT and brane quantum gravity}

Let us start from the discussion of AdS/CFT correspondence (on the level of holographic conformal anomaly). We argue that AdS/CFT set-up opens the window for brane quantum gravity as dual theory. In its own turn, this indicates on even better understanding of Randall-Sundrum compactification [10] within AdS/CFT correspondence [1] in string theory.

Let us start from $d+1$ dimensional gravity on AdS background. The action is given by

$$
S=\int d^{d+1} x \sqrt{-\hat{g}}\left\{\frac{1}{16 \pi G} \hat{R}-\Lambda\right\}
$$

The AdS metric may be chosen as follows

$$
d s^{2}=\frac{l^{2}}{4} \rho^{-2} d \rho d \rho+\sum_{i=1}^{d} \rho^{-1} g_{\mu \nu} d x^{\mu} d x^{\nu}
$$

\footnotetext{
${ }^{3}$ For earlier discussion of entropy in expanding Universe, see, for example[9.
} 
where $g_{\mu \nu}$ is metric of boundary manifold, and dimensional parameter $l$ is introduced explicitly. Note that dimensional parameter is related with bulk cosmological constant:

$$
l^{2}=-\frac{d(d-1)}{16 \pi G \Lambda}
$$

as it is dictated by Eq. of motion. Using several methods (holographic RG or expansion of classical AdS action in powers of a cut-off parameter), one can get the holographic conformal anomaly (CA). This CA should correspond to boundary dual QFT.

Explicitly, for $d=2$ :

$$
T=-\frac{l}{\kappa^{2}} R, \quad \kappa^{2}=16 \pi G
$$

For $d=4$ :

$$
T=-\frac{l^{3}}{8 \kappa^{2}}(G-F)
$$

where $G=R^{2}-4 R_{\mu \nu} R^{\mu \nu}+R_{\mu \nu \rho \sigma} R^{\mu \nu \rho \sigma}, F=\frac{1}{3} R^{2}-2 R_{\mu \nu} R^{\mu \nu}+R_{\mu \nu \rho \sigma} R^{\mu \nu \rho \sigma}$ and $R$ are $d$-dimensional curvature invariants for $d+1$-dimensional theory (11).

The next step is comparison with dual QFT CA. Explicit calculation gives

$$
T=b F+b^{\prime}\left(G-\frac{2}{3} \square R\right)
$$

where

$$
\begin{aligned}
b & =\frac{N+6 N_{1 / 2}+12 N_{1}-8 N_{\mathrm{HD}}+611 N_{2}+796 N_{W}}{120(4 \pi)^{2}} \\
b^{\prime} & =-\frac{N+11 N_{1 / 2}+62 N_{1}-28 N_{\mathrm{HD}}+1411 N_{2}+1566 N_{W}}{360(4 \pi)^{2}} .
\end{aligned}
$$

Here $N, N_{1 / 2}, N_{1}, N_{\mathrm{HD}}$ is the number of scalars, (Dirac) spinors, vectors and higher derivative conformal scalars which present in boundary QFT. $N_{2}$ denotes the contribution to CA from spin-2 field (Einstein gravity) and $N_{W}$ the contribution from higher derivative Weyl gravity. (For the calculation of quantum gravity corrections to CA see [11]). Note also that $\square R$-term in $4 \mathrm{~d}$ $\mathrm{CA}$ is ambigious as it may depend on the regularization choice. Moreover, it may be changed by finite renormalization of classical gravitational action. That is why this term is of no interest in the present context. 
It is clear that for AdS/CFT correspondence on the level of CA, two expressions (5) and (6) (when no $\square R$-term presents) should coincide. To find the corresponding QFT dual one has the condition:

$$
b=-b^{\prime} .
$$

In pure matter sector there is following natural possibility:

$$
N=6 c, \quad N_{\frac{1}{2}}=2 c, \quad N_{1}=c
$$

where $c$ is an arbitrary number. The choice of $c=N^{2}-1$ gives $\mathcal{N}=4$ $S U(N)$ super Yang-Mills theory multiplet which is known to be conformally invariant theory where

$$
b=\frac{N^{2}-1}{4(4 \pi)^{2}} .
$$

This is standard AdS/CFT correspondence on the level of CAs. The AdS/CFT choice

$$
\frac{l^{3}}{8 \kappa^{2}}=\frac{N^{2}}{4(4 \pi)^{2}}
$$

leads to coincidence of holographic conformal anomaly with QFT CA in the leading order of large- $N$.

The remark is in order. The choice (9) is not unique. For example, the condition (8) is fulfilled for

$$
\begin{array}{lll}
N_{1}=2 c_{1}, & N_{\frac{1}{2}}=2, & N=12 \\
N_{1}=2 c_{1}, & N_{\frac{1}{2}}=4, & N=12 \\
N_{1}=2 c_{1}, & N_{\frac{1}{2}}=6, & N=5 .
\end{array}
$$

Increasing the number of vectors leads to more variants for scalar and spinors. Of course, such theories are only conformally invariant ones as free theories (no interaction). It is very interesting that condition (9) in matter sector looks as

$$
2 N+7 N_{\frac{1}{2}}=26 N_{1} .
$$

This relation appears in the study of stability of Nariai BH. As it was shown in [12], if conformal GUT matter content satisfies $2 N+7 N_{\frac{1}{2}}>26 N_{1}$ the $4 \mathrm{~d}$ Nariai BH is stable (in 4d Einstein gravity with quantum corrections due to 
such GUT). No BH anti-evaporation occurs. It is also interesting that not only SUSY theories satisfy the bound (13), but also non-SUSY ones. That suggests the possibility of other duals for d5 AdS Einstein gravity.

What is more interesting, one can take into account the brane QG in relation (8). The easy check shows that considering Einstein brane gravity $\left(N_{2}=1, N_{W}=0\right)$ with scalars, spinors and vectors, the relation (8) holds for Einstein gravity with $\left(N_{1}=17, N_{\frac{1}{2}}=0, N=10\right),\left(N_{1}=17, N_{\frac{1}{2}}=2\right.$, $N=3),\left(N_{1}=18, N_{\frac{1}{2}}=2, N=16\right),\left(N_{1}=18, N_{\frac{1}{2}}=4, N=9\right),\left(N_{1}=18\right.$, $\left.N_{\frac{1}{2}}=6, N=2\right)$. With increase of vector number, the number of choice for scalars and spinors increases as well. Similarly, it is easy to check that 4d Weyl gravity with some matter content gives QFT anomaly reproducing holographic anomaly. This indicates to possible AdS/CFT duality between bulk d5 AdS gravity and brane quantum (Einstein or Weyl) gravity with some (non)-supersymmetric amount of matter. Of course, other checks should be considered in such proporsal as only comparison of anomalies is not enough. However, it gives useful indication on dual brane QG for d5 bulk gravity!

Moreover, various scenarios can be suggested. For example, QG with matter may be subdominant. It gives the contribution to $b$ of about $\frac{7}{(4 \pi)^{2}}$. Then if $N>7$, the dual QFT may be $\mathcal{N}=4 S U(N)$ super Yang-Mills theory with (Einstein) QG with matter. The Einstein gravity with (nonsupersymmetric) matter will correspond to next-to-leading order of large $N$ expansion. Other variants of such scenario may be considered as well.

In $d=2$ cases the comparison is even easier. QFT CA is equal here:

$$
T=\frac{c R}{2 \pi},
$$

where $c=N+N_{\frac{1}{2}}-25$. The last term is contribution of d2 Einstein gravity. As one sees it is again easy to achieve the coincidence of holographic CA (7) with QFT plus brane QG result via the identification of $\frac{l}{\kappa^{2}}$ and $\frac{c}{2 \pi}$. It again suggests on the appearence of dual brane QG with matter in $\mathrm{AdS}_{3} / \mathrm{CFT}_{2}$ correspondence.

Thus, we presented the arguments which indicate that dual brane QG (non-conformal Einstein or conformal Weyl one) may also appear in AdS/CFT correspondence. Of course, more work is necessary to understand better the appearence of brane QG on dual QFT side of AdS/CFT correspondence. However, the indications we presented here look quite promising. 


\section{AdS/CFT and quantum induced 3d brane- worlds}

It is expected that AdS/CFT correspondence [1] should be related with Randall-Sundrum orbifold compactification [10] which presumbly is realized as warped compactification in string theory. One scenario of this sort has been suggested in refs. [4, 5] where the quantum effects of brane CFT (including brane QG) have been taking into account. In this way 4d New Brane World may be constructed in frames of AdS/CFT set-up (as a kind of holographic renormalization group flow). Moreover, such scenario is extended now for d5 gauged supergravity (inclusion of non-constant dilaton) where non-singular dilatonic inflationary brane-world occurs[7] (for related works, see 13, 15, 14). The important role in the construction of such brane quantum field theory induced bulk Universe belongs to conformal anomaly. In the present section using $2 \mathrm{~d}$ conformal anomaly we demonstrate the universality of scenario [由, 5] in various dimensions. In particulary, we show that it works also for 3d inflationary (or hyperbolic) brane-worlds in AdS3/CFT2 correspondence.

We start with the action $S$ which is the sum of the three-dimensional Einstein-Hilbert action $S_{\mathrm{EH}}$, the Gibbons-Hawking surface term $S_{\mathrm{GH}}$, the surface counter term $S_{1}$ and the $2 \mathrm{~d}$ trace anomaly induced action $W$ :

$$
\begin{aligned}
S & =S_{\mathrm{EH}}+S_{\mathrm{GH}}+2 S_{1}+W \\
S_{\mathrm{EH}} & =\frac{1}{\kappa^{2}} \int d^{3} x \sqrt{g_{(3)}}\left(R_{(3)}-\frac{2}{l^{2}}\right) \\
S_{\mathrm{GH}} & =\frac{2}{\kappa^{2}} \int d^{2} x \sqrt{g_{(2)}} \nabla_{\mu} n^{\mu} \\
S_{1} & =\frac{2}{\kappa^{2}} \int d^{2} x \sqrt{g_{(2)}} \\
W & =-\frac{1}{2} \int d^{2} x \sqrt{-g_{(2)}} \frac{N}{48 \pi} R_{(2)} \frac{1}{\Delta_{(2)}} R_{(2)} .
\end{aligned}
$$

A solution in the bulk 3d spacetime is anti-de Sitter (AdS) space, whose metric is given by

$$
d s^{2}=d z^{2}+\mathrm{e}^{\frac{2 z}{l}} \sum_{i=1}^{2}\left(d x^{i}\right)^{2}
$$


In (20) the slice of constant $z$ is a flat $2 \mathrm{~d}$ space. One can choose, however, so that the slice is $2 \mathrm{~d}$ sphere or hyperboloid.

$$
\begin{aligned}
& d s^{2}=d z^{2}+l^{2} \sinh ^{2} \frac{z}{l} d S_{2}^{2} \\
& d s^{2}=d z^{2}+l^{2} \cosh ^{2} \frac{z}{l} d H_{2}^{2} .
\end{aligned}
$$

Here $d S_{2}^{2}$ and $d H_{2}^{2}$ expresses the metric of unit radius $2 \mathrm{~d}$ sphere and hyperboloid, respectively. The metrics $d S_{2}^{2}$ and $d H_{2}^{2}$ can be, for example, expressed in the following forms:

$$
d S_{2}^{2}=\frac{1}{\cosh ^{2} \sigma}\left(d \sigma^{2}+d \phi^{2}\right), \quad d H_{2}^{2}=\frac{1}{\sinh ^{2} \sigma}\left(d \sigma^{2}+d \phi^{2}\right) .
$$

Here $\phi$ has a period of $2 \pi$. The metric of the flat $2 \mathrm{~d}$ space is also expressed as

$$
\sum_{i=1}^{2} d x_{i}^{2}=l^{2} \mathrm{e}^{2 \sigma}\left(d \sigma^{2}+d \phi^{2}\right) .
$$

Then all metrics in (20) and (21) have the following common form:

$$
d s^{2}=d z^{2}+l^{2} \mathrm{e}^{A(z, \sigma)}\left(d \sigma^{2}+d \phi^{2}\right), \quad A(z, \sigma)=A_{1}(z)+A_{2}(\sigma) .
$$

We now assume that there is a brane at $z=z_{0}$. The shape of the brane can be $2 \mathrm{~d}$ sphere $\mathrm{S}_{2}, 2 \mathrm{~d}$ flat space $\mathrm{R}_{2}$ or $2 \mathrm{~d}$ hyperboloid $\mathrm{H}_{2}$ by the choice of the metric. Then by the variation of the action with respect to $A$, one obtains the following equations on the boundary:

$$
\frac{8}{\kappa^{2}}\left(-A_{1, z}\left(z_{0}\right)+\frac{1}{l}\right)+\frac{N}{12 \pi} k \mathrm{e}^{A_{1}\left(z_{0}\right)}=0 .
$$

Here $k=1$ for $\mathrm{S}_{2}, k=0$ for $\mathrm{R}_{2}$ and $k=-1$ for $\mathrm{H}_{2}$. Note that $A_{1}(z)=$ $\ln \cosh \frac{z}{l}$ for $\mathrm{S}_{2}, A_{1}(z)=\frac{z}{l}$ for $\mathrm{R}_{2}$ and $A_{1}(z)=\ln \sinh \frac{z}{l}$ for $\mathrm{H}_{2}$. For $2 \mathrm{~d}$ flat space, Eq.(26) becomes identity. For $2 \mathrm{~d}$ sphere, Eq.(26) has the following form:

$$
\frac{8}{\kappa^{2} l}\left(\operatorname{coth} \frac{z_{0}}{l}-1\right) \sinh ^{2} \frac{z_{0}}{l}=\frac{N}{12 \pi}
$$

and for 2d hyperboloid, Eq.(26) has the following form:

$$
\frac{8}{\kappa^{2} l}\left(1-\tanh \frac{z_{0}}{l}\right) \cosh ^{2} \frac{z_{0}}{l}=\frac{N}{12 \pi} .
$$


Both of the equations (27) and (28) can be uniquely solved with respect to $z_{0}$. This situation is different from the situation in $4 \mathrm{~d}$ quantum induced curved brane 41 in $\mathrm{AdS}_{5}$, where there is no usually solution for the brane of the shape of the hyperboloid. The difference appears since the trace anomaly is linear on the scalar curvature in 2 dimensions but the anomaly is proportional to the squares of the curvatures in 4 dimensions. In 2 dimensions, the change of the sign coming from $k$ can be absorbed into the change of sign in the scalar curvature (the scalar curvature is positive for $2 \mathrm{~d}$ sphere but negative for hyperboloid). Since the radius $R$ of the brane can be defined by

$$
R=l \mathrm{e}^{A_{1}\left(z_{0}\right)},
$$

Eqs.(27) and (28) can be rewritten in the following forms, respectively:

$$
\begin{aligned}
& \frac{8 R}{\kappa^{2} l^{2}}\left(\sqrt{\frac{R^{2}}{l^{2}}+1}-\frac{R}{l}\right)=\frac{N}{12} \\
& \frac{8 R}{\kappa^{2} l^{2}}\left(\frac{R}{l}-\sqrt{\frac{R^{2}}{l^{2}}-1}\right)=\frac{N}{12} .
\end{aligned}
$$

Both of the l.h.s.'s in Eqs.(30) and (31) are monotonically increasing functions. Since the l.h.s. in $(30)$ vanishes when $\mathcal{R}=0$ and goes to positive infinity when $\mathcal{R} \rightarrow \infty$, (30) determines $\mathcal{R}$ uniquely. On the other hand, the l.h.s. in (31) becomes $\frac{8}{\kappa^{2} l}$ when $R=l$. Therefore if

$$
\frac{8}{\kappa^{2} l}<\frac{N}{12}
$$

there is a non-trivial solution of the hyperbolic brane. Thus, we demonstrated the possibility of inflationary or hyperbolic $3 \mathrm{~d}$ brane-world induced by quantum effects of brane matter. As we saw in previous section the $2 \mathrm{~d}$ conformal anomaly may include also quantum contribution from $2 \mathrm{~d}$ gravity (then sign of quantum effective action $W$ may be positive, depending on the amount of matter). This indicates that scenario of refs. [4, 5] is quite universal. Moreover, in the same way as in ref. [7] it may be easily extended for the presence of non-trivial dilaton(s). 


\section{Quantum-corrected energy and quantum entropy bounds in FRW-Universe}

Holographic principle suggests the interesting bounds between microscopic entropy and Bekenstein-Hawking entropy [16] as it was suggested in refs. [17]. This indicates also to the relations between AdS/CFT set-up and entropy and brings new bounds for the entropy [18, 19, 20] (for related works on holographic entropy, see 21]). Note that it would be really interesting to understand the entropy bounds origin from stringy (AdS/CFT) points of view (for recent attempt to understand cosmology/CFT set-up via corresponding comparison of entropies, see [22]).

In the present section we make the attempt to understand the role of (large) quantum corrections to energy/entropy (using again the conformal anomaly ) in the study of entropy bounds and their dynamical evolution [8]( for related works, see [23]). The dynamical equations describing FRWcosmology and energy/entropy are defined in the presence of quantum corrections which could be dominant ones. Our analysis suggests that the effect of (large) quantum contribution may destroy the well-accepted entropy bounds which become the approximate ones and which occur only for some timelimited period of Universe evolution.

We start with the following action:

$$
\begin{aligned}
S= & \frac{1}{16 \pi G} \int d^{4} x \sqrt{-g} R_{(4)}+S_{\text {matter }}+W \\
W= & b \int d^{4} x \sqrt{-\widetilde{g}} \widetilde{F} A \\
& +b^{\prime} \int d^{4} x \sqrt{-\widetilde{g}}\left\{A\left[2 \square^{2}+\widetilde{R}_{\mu \nu} \widetilde{\nabla}_{\mu} \widetilde{\nabla}_{\nu}-\frac{4}{3} \widetilde{R} \square^{2}+\frac{2}{3}\left(\widetilde{\nabla}^{\mu} \widetilde{R}\right) \widetilde{\nabla} \mu\right] A\right. \\
& \left.+\left(\widetilde{G}-\frac{2}{3} \square \widetilde{R}\right) A\right\} \\
& -\frac{1}{12}\left\{b^{\prime \prime}+\frac{2}{3}\left(b+b^{\prime}\right)\right\} \int d^{4} x \sqrt{-\widetilde{g}}\left[\widetilde{R}-6 \square A-6\left(\widetilde{\nabla}_{\mu} A\right)\left(\widetilde{\nabla}^{\mu} A\right)\right]^{2} \\
& +S_{\text {inv }} .
\end{aligned}
$$

The last term $(W)$ represents the conformal anomaly induced effective action (for a review, see [24]). Here one chooses the metric in the following form:

$$
d s^{2}=\mathrm{e}^{2 A(\tau)} \tilde{g}_{\mu \nu} d x^{\mu} d x^{\nu} \equiv \mathrm{e}^{2 A(\tau)}\left(-d \tau^{2}+d \Omega_{3}^{2}\right)
$$


$d \Omega_{3}^{2}$ expresses the metric of 3 dimensional sphere. We denote the $A$ independent terms by $S_{\text {inv }}$ which would be given as

$$
S_{\text {Inv }}=\int d^{4} x \sqrt{-\widetilde{g}}\left(a_{1} \widetilde{R}^{2}+a_{2} \widetilde{R}_{\mu \nu} \widetilde{R}^{\mu \nu}+a_{3} \widetilde{R}_{\mu \nu \rho \sigma} \widetilde{R}^{\mu \nu \rho \sigma}\right)+\text { non-local terms }
$$

It is clear that such terms (Casimir energy of static space) cannot be obtained by only the integration of conformal anomaly.

Let us neglect the non-local terms in (35) and choose the metric in (34) as (35) then

$$
S_{\text {Inv }}=\tilde{a} \int d^{4} x \sqrt{\widetilde{g}}, \quad \tilde{a}=36 a_{1}+12 a_{2}+12 a_{3} .
$$

By the variation over $\tilde{g}_{\tau \tau}$, we obtain the following equation (conservation law):

$$
\begin{aligned}
0= & \frac{1}{16 \pi G} \mathrm{e}^{2 A}\left\{6+6\left(\partial_{\tau} A\right)^{2}\right\}+2 \mathrm{e}^{6 A} T_{\text {matter }}^{\tau \tau} \\
& +b^{\prime}\left\{4 \partial_{\tau} A \partial_{\tau}^{3} A-2\left(\partial_{\tau}^{2} A\right)^{2}+8\left(\partial_{\tau} A\right)^{2}\right\} \\
& -\frac{1}{12}\left\{b^{\prime \prime}+\frac{2}{3}\left(b+b^{\prime}\right)\right\}\left\{-36\left(\partial_{\tau}^{2} A\right)^{2}-108\left(\partial_{\tau} A\right)^{4}\right. \\
& \left.+72 \partial_{\tau} A \partial_{\tau}^{3} A-72\left(\partial_{\tau} A\right)^{2}+36\right\}+\tilde{a}
\end{aligned}
$$

Here $T^{\tau \tau}$ is the stress energy tensor of the matter

$$
T^{\tau \tau}=-\frac{2}{\sqrt{-g}} \frac{\delta S_{\mathrm{matter}}}{\delta g_{\tau \tau}}
$$

On the other hand, varying over $A$ one gets the dynamical field equation

$$
\begin{aligned}
0= & \frac{1}{16 \pi G} \mathrm{e}^{2 A}\left\{12+12 \partial_{\tau}^{2} A+12\left(\partial_{\tau} A\right)^{2}\right\} \\
& +b^{\prime}\left\{4 \partial_{\tau}^{4} A+16 \partial_{\tau}^{2} A\right\} \\
& -\left\{b^{\prime \prime}+\frac{2}{3}\left(b+b^{\prime}\right)\right\}\left\{6 \partial_{\tau}^{6} A-36\left(\partial_{\tau} A\right)^{2} \partial_{\tau}^{2} A-12 \partial_{\tau}^{2} A\right\}
\end{aligned}
$$

Our assumption is the matter is conformally invariant, that is, its classical stress-energy tensor is traceless. Then the matter does not contribute to the 
above equation (39). We now change the time coordinate from the conformal one $\tau$ to the cosmological one $t$ by

$$
d t=\mathrm{e}^{A} d \tau .
$$

One also defines the radius of the universe $R$ and the Hubble constant $H$ as follows:

$$
R \equiv \mathrm{e}^{A}, \quad H=\frac{1}{R} \frac{d R}{d t}=\frac{d A}{d t} .
$$

Then Eqs.(37) and (39) are rewritten as follows:

$$
\begin{aligned}
H^{2}= & -\frac{1}{R^{2}}+\frac{16 \pi G}{6} \frac{E}{V} \\
\frac{E}{V} \equiv T^{t t}-b^{\prime}\left(4 H H_{, t t}+12 H_{, t} H^{2}-2 H_{, t}^{2}+6 H^{4}+\frac{8}{R^{2}} H^{2}\right) & +\frac{1}{12}\left\{b^{\prime \prime}+\frac{2}{3}\left(b+b^{\prime}\right)\right\} \\
& \times\left(-36 H_{, t}^{2}+216 H_{, t} H^{2}+72 H H_{, t t}-\frac{72}{R^{2}} H^{2}+\frac{36}{R^{4}}\right) \\
& +\frac{\tilde{a}}{R^{4}}, \\
0 & \frac{1}{16 \pi G}\left(\frac{12}{R^{2}}+24 H^{2}+12 H_{, t}\right) \\
& +b^{\prime}\left\{4 H_{, t t t}+28 H H_{, t t}+16 H_{, t}^{2}+72 H^{2} H_{, t}\right. \\
& \left.+24 H^{4}+\frac{16}{R^{2}}\left(H_{, t}+H^{2}\right)\right\} \\
& -\left\{b^{\prime \prime}+\frac{2}{3}\left(b+b^{\prime}\right)\right\}\left\{6 H_{, t t t}+42 H H_{, t t}+24 H_{, t}^{2}+72 H^{2} H_{, t}\right. \\
& \left.-\frac{12}{R^{2}}\left(H_{, t}+H^{2}\right)\right\} .
\end{aligned}
$$

Here

$$
T^{t t}=\mathrm{e}^{2 A} T^{\tau \tau} .
$$

Especially when $b=b^{\prime}=b^{\prime \prime}=0$, the equation (44) has the following form:

$$
0=\frac{12}{R^{2}}+24 H^{2}+12 H_{, t} .
$$


If one deletes $H^{2}$ term from (46) by using (42), then

$$
H_{, t}=\frac{1}{R^{2}}-\frac{16 \pi G}{3} \frac{E}{V},
$$

This is the standard evolution equation of Einstein theory for the conformal matter, where $3 p=E$ ( $p$ is the pressure). The important remark is in order. In the above formalism the matter is considered to be classical and only classical stress-tensor appears. The quantum effects of matter (and of quantum gravity) via the corresponding anomaly induced effective action $W$ modify the classical general relativity by extra (non-local) terms. Hence, quantum effects are accounted here via the modification of classical gravity.

If one defines the Bekenstein-Hawking entropy $\mathcal{S}_{\mathrm{BH}}$, the Bekenstein entropy $\mathcal{S}_{\mathrm{B}}$ and the Hubble entropy $\mathcal{S}_{\mathrm{H}}$ as

$$
\mathcal{S}_{\mathrm{BH}}=\frac{1}{2} \frac{V}{G R}, \quad \mathcal{S}_{\mathrm{B}}=\frac{2}{3} \pi E R, \quad \mathcal{S}_{\mathrm{H}}=\frac{2 H V}{4 G},
$$

Eq.(42) leads to

$$
\mathcal{S}_{\mathrm{H}}^{2}+\left(\mathcal{S}_{\mathrm{BH}}-\mathcal{S}_{\mathrm{B}}\right)^{2}=\mathcal{S}_{\mathrm{B}}^{2}
$$

When $T^{t t}=0$, the solution in the form of de Sitter space exists [6, 25]

$$
R=A \cosh B t, \quad A, B=\text { constant }
$$

Using (50) in (42) and (43), we find that (50) is solution if

$$
B^{2}=\frac{1}{A^{2}}=-\frac{1}{16 \pi G b^{\prime}} .
$$

This is the basis for anomaly driven inflation [6] ( for recent extended arguments in favor of realistic anomaly-driven inflation ,see [15]). When one substitutes Eqs.(50) and (51), Eq. (42) is satisfied if

$$
\tilde{a}=-8 b^{\prime} \text {. }
$$

In the following, we assume $\tilde{a}$ is given by (52) (the choice of normalization). The obtained metric of de Sitter space has the following form:

$$
d s^{2}=-d t^{2}+A^{2} \cosh ^{2} \frac{t}{A} d \Omega_{3}^{2}
$$


If we analytically continue $t$ as

$$
t=i A\left(\theta-\frac{\pi}{2}\right)
$$

we obtain the metric of 4 dimensional sphere $\mathrm{S}_{4}$ :

$$
d s^{2}=A^{2}\left(d \theta^{2}+\sin ^{2} \theta d \Omega_{3}^{2}\right)
$$

Identifying the north pole $(\theta=0)$ and the south pole $(\theta=\pi)$, the period of $\theta$ is $\pi$. Then one could regard that $t$ has a period $\frac{\pi}{A}$, which is related with the inverse temperature $\mathcal{T}$

$$
\mathcal{T}=\frac{A}{\pi}
$$

In the above discussion we used the approach where quantum effects modify the gravitational effective action. One can show that it is equivalent to the approach where quantum corrections to stress-energy tensor are considered. Indeed in [15] there has been given an elegant method to obtain the (quantum corrected) energy in the spacetime with the metric:

$$
d s^{2}=d \sigma^{2}+b(\sigma)^{2} d \Omega_{3}^{2} .
$$

The authors of ref. 15 worked in the Euclidean metric in order to consider instanton solutions. Then one can identify

$$
\sigma=i t, \quad b(\sigma)=R(t)
$$

Here $t$ and $R(t)$ were given in (40) and (43), respectively. In the following, we write $b(\sigma)$ as $R$ in order to avoid the confusion with $b, b^{\prime}$ and $b^{\prime \prime}$ in (33) and only consider the Lorentzian signature case. In [15], there was found the quantum energy for $\mathcal{N}=4 S U(N)$ or $U(N)$ supersymmetric Yang-Mills (SYM) theory, where

$$
b=-b^{\prime}=\frac{N^{2}}{4(4 \pi)^{2}}, \quad b^{\prime \prime}=0,
$$

Here we generalize the method to more general $b, b^{\prime}$ and $b^{\prime \prime}$ case. It is interesting that formally one can include the quantum gravity corrections to coefficients of conformal anomaly (see Section 2), then quantum stress-energy 
tensor is given also by sum of two parts: matter and gravity. We now define the energy density $\rho$ and pressure $p$ by

$$
\left\langle T_{t t}\right\rangle=\rho, \quad\left\langle T_{i j}\right\rangle=p g_{i j}=p \mathrm{e}^{2 A} \tilde{g}_{i j}(i, j=1,2,3),
$$

Then

$$
\langle T\rangle \equiv g^{\mu \nu}\left\langle T_{\mu \nu}\right\rangle=-\rho+3 p
$$

On the other hand, the conservation law of the energy-momentum tensor $\nabla^{\mu} T_{\mu \nu}=0$ tells

$$
0=\rho_{, t}+3 A_{, t}(\rho+p) .
$$

Combining (61) and (62), one gets

$$
\left(\mathrm{e}^{4 A} \rho\right)_{, t}=-\mathrm{e}^{4 A} A_{, t}\langle T\rangle .
$$

Since now the trace anomaly is given by

$$
\begin{aligned}
\langle T\rangle= & b\left(F+\frac{2}{3} \square \mathcal{R}\right)+b^{\prime} G+b^{\prime \prime} \square \mathcal{R} \\
= & b^{\prime} \mathrm{e}^{-3 A}\left\{8\left(\mathrm{e}^{3 A} A_{, t}^{3}\right)_{, t}+24\left(\mathrm{e}^{A} A_{, t}\right)_{, t}\right\} \\
& -\left(\frac{2}{3} b+b^{\prime \prime}\right) \mathrm{e}^{-3 A}\left\{\mathrm{e}^{3 A}\left(6 A_{, t t}+12 A_{, t}^{2}+6 \mathrm{e}^{-2 A}\right)_{, t}\right\}_{, t},
\end{aligned}
$$

one finds

$$
\begin{aligned}
\rho= & -\frac{1}{R^{4}}\left[b^{\prime}\left(6 R^{4} H^{4}+12 R^{2} H^{2}\right)\right. \\
& \left.+\left(\frac{2}{3} b+b^{\prime \prime}\right)\left\{R^{4}\left(-6 H H_{, t t}-18 H^{2} H_{, t}+3 H_{, t}^{2}\right)+6 R^{2} H^{2}\right\}+C\right]
\end{aligned}
$$

Here $C$ is the integration constant. If we choose the constant $C$ as

$$
C=-3 b^{\prime \prime}-2\left(b+b^{\prime}\right)-\tilde{a}=-2 b+6 b^{\prime}-3 b^{\prime \prime},
$$

we find that $\rho$ coincides with $\frac{E}{V}$ in $(43)$ when there is no contribution from the classical matter $\left(T_{t t}=0\right)$. This proves the equivalency of two formalisms to derive the evolution equations. 
One can also obtain the expression of the pressure $p$ from (61), (64) and (65):

$$
\begin{aligned}
p= & b^{\prime}\left\{6 H^{4}+8 H^{2} H_{, t}+\frac{1}{R^{2}}\left(4 H^{2}+8 H_{, t}\right)\right\} \\
& +\left(\frac{2}{3} b+b^{\prime \prime}\right)\left\{-2 H_{, t t t}-12 H H_{, t t}-18 H^{2} H_{, t}-9 H_{, t}^{2}\right. \\
& \left.+\frac{1}{R^{2}}\left(2 H^{2}+4 H_{, t}\right)\right\}-\frac{C}{3 R^{4}} .
\end{aligned}
$$

One can now divide $\frac{E}{V}$ in (43) or $\rho$ in (65) and $p$ in (67) with the contribution from the classical matter by the $R$ dependence as follows

$$
\begin{aligned}
\rho= & \rho_{0}+\rho_{1}+\rho_{2} \\
\rho_{0}= & T_{t t}-6 b^{\prime} H^{4}-\left(\frac{2}{3} b+b^{\prime \prime}\right)\left(-6 H H_{, t t}-18 H^{2} H_{, t}+3 H_{, t}^{2}\right) \\
\rho_{1}= & -\left\{12 b^{\prime}+6\left(\frac{2}{3} b+b^{\prime \prime}\right)\right\} \frac{H^{2}}{R^{2}} \\
\rho_{2}= & -\frac{C}{R^{4}} \\
p= & p_{0}+p_{1}+p_{2} \\
p_{0}= & \frac{1}{3} T_{t t}+b^{\prime}\left(6 H^{4}+8 H^{2} H_{, t}\right) \\
& +\left(\frac{2}{3} b+b^{\prime \prime}\right)\left(-2 H_{, t t t}-12 H H_{, t t}-18 H^{2} H_{, t}-9 H_{, t}^{2}\right) \\
p_{1}= & \frac{1}{R^{2}}\left\{b^{\prime}\left(4 H^{2}+8 H_{, t}\right)+\left(\frac{2}{3} b+b^{\prime \prime}\right)\left(2 H^{2}+4 H_{, t}\right)\right\} \\
p_{2}= & -\frac{C}{3 R^{4}} .
\end{aligned}
$$

$\rho_{0}$ does not depend on $R$, therefore $\rho_{0}$ expresses the extensive part of the energy. On the other hand, $\rho_{1}$ and $\rho_{2}$ will express the finite size effects (like the Casimir energy). Using the thermodynamical equation for the entropy $\mathcal{S}$

$$
E=-p V+\mathcal{T S}
$$

we naively find the entropy $\mathcal{S}$ is given by

$$
\mathcal{S}=\frac{V(\rho+p)}{\mathcal{T}}
$$


It is very important to realize that quantum contributions in above expression (as well as for energy) may be dominant if compare with classical expression!

Let us consider the case of the de Sitter space solution in (53) with (51). In this case, the quantum energy density $\rho$ (65) and the quantum pressure $p$ (67) are given by

$$
\rho=-p=-\frac{6 b^{\prime}}{A^{4}}-6 b^{\prime} B^{4}=-\frac{6}{(16 \pi G)^{2} b^{\prime}} .
$$

This indicates that quantum entropy $\mathcal{S}$ vanishes (from (71)) if the temperature is finite as in (56). This might tell that the definition of the entropy (if the temperature $\mathcal{T}$ is defined) is not correct. Usually the entropy is extensive quantity, i.e., proportional to the volume of the space. Eqs. (68) and (69) might, however, tell that the definition in (71) is a mixture of the extensive part and non-extensive part.

In [8], another better way to define the entropy, which is extensive, has been proposed. The energy $E=\rho V$ is divided to the extensive part $E_{E}$ and the Casimir (or quantum) part $E_{C}$ :

$$
E=E_{E}+\frac{1}{2} E_{C}
$$

If one introduces a length parameter $l, E_{E}$ scales as $E_{E} \sim l^{3}$ and $E_{C}$ as $E_{C} \sim l$. If there is a conformal symmetry, both of $R E_{E}$ and $R E_{C}$ should only depend on the entropy $\mathcal{S}$. If the entropy $\mathcal{S}$ is extensive quantity and scales as $\mathcal{S} \sim l^{3}$, one finds

$$
R E_{E} \propto \mathcal{S}^{\frac{4}{3}}, \quad R E_{C} \propto \mathcal{S}^{\frac{2}{3}} .
$$

Therefore

$$
\mathcal{S} \propto \sqrt{E_{E} E_{C}} .
$$

In [8], the constant of the proportionality has been determined from the AdS/CFT correspondence as

$$
\mathcal{S}=\frac{2 \pi R}{3} \sqrt{2 E_{E} E_{C}} .
$$

By using (68), we might be able to identify

$$
E_{E}=\rho_{0} V, \quad E_{C}=2 \rho_{1} V
$$


and we have

$$
\mathcal{S}=\frac{4 \pi R V}{3} \sqrt{\rho_{0} \rho_{1}} .
$$

Here we dropped the integration constant dependent $\rho_{2}$ because the entropy would not become extensive if we include $\rho_{2}$ since $\rho_{2}$ is expected to scale like $l^{-1}$. Since $b^{\prime \prime}$ can be shifted by the finite regularization, we choose $b^{\prime \prime}=-\frac{2}{3} b$ for simplicity. Then from (68), one gets

$$
\mathcal{S}=-8 \pi \sqrt{2} V b^{\prime} H^{3} .
$$

Here we put the energy density of the classical matter to vanish $T_{t t}=0$. We now consider the solution of the de Sitter space in (53) with (51) and compare the entropy in (79) with the entropies defined in (48). Using de Sitter solution in the expression for the entropies, one gets

$$
\begin{aligned}
\mathcal{S} & =-8 \pi \sqrt{2} V b^{\prime} B^{3} \tanh ^{3} B t \\
\mathcal{S}_{B H} & =-\frac{8 \pi V b^{\prime} B^{3}}{\cosh B t} \\
\mathcal{S}_{B} & =-4 \pi V b^{\prime} B^{3} \cosh B t \\
\mathcal{S}_{H} & =-8 \pi V b^{\prime} B^{3} \tanh B t .
\end{aligned}
$$

In (80), $\mathcal{S}, \mathcal{S}_{\mathcal{B}}$ and $\mathcal{S}_{H}$ are monotonicaly increasing functions of $t$ when $t \geq 0$. $\mathcal{S}$ and $\mathcal{S}_{H}$ vanish and $\mathcal{S}_{B}$ has a minimum value $-4 \pi V b^{\prime} B^{3}$ when $t=0$ and $\mathcal{S}$ and $\mathcal{S}_{H}$ take a maximum values, $-8 \pi \sqrt{2} V b^{\prime} B^{3}$ and $-8 \pi V b^{\prime} B^{3}$, respectively, and $\mathcal{S}_{B}$ increases exponentially when $t \rightarrow+\infty$. On the other hand, $\mathcal{S}_{B H}$ is a monotonically decreasing function of $t$ when $t \geq 0$. $\mathcal{S}_{B H}$ takes a maximum value $-8 \pi V b^{\prime} B^{3}$ when $t=0$ and vanishes when $t \rightarrow+\infty$. $\mathcal{S}_{B}, \mathcal{S}_{\mathcal{H}}$ and $\mathcal{S}_{B H}$ coincide with each other when $\tanh B t=\tanh B t_{1}=\frac{1}{\sqrt{2}}=0.7071 \ldots$. One finds $\mathcal{S}_{\mathcal{B H}}>\mathcal{S}_{B}>\mathcal{S}_{H}$ when $t<t_{1}$ and $\mathcal{S}_{B}>\mathcal{S}_{H}>\mathcal{S}_{\mathcal{B H}}$ when $t>t_{1}$. We should note that $\mathcal{S}_{B}$ does not cross with $\mathcal{S}_{H}$ but $\mathcal{S}_{B}$ is tangent to $\mathcal{S}_{H}$ when $t=t_{1}$. $\mathcal{S}$ crosses with $\mathcal{S}_{B H}$ when $\tanh B t=\tanh B t_{2}=2^{-\frac{1}{4}}=0.7679 \ldots$ and with $\mathcal{S}_{H}$ when $\tanh B t=\tanh B t_{3}=0.84089 \ldots$. By summarizing the above behavior of the entropies, one gets

$$
\begin{array}{ll}
\mathcal{S}_{\mathcal{B H}}>\mathcal{S}_{B}>\mathcal{S}_{H}>\mathcal{S} & t<t_{1} \\
\mathcal{S}_{B}>\mathcal{S}_{H}>\mathcal{S}_{\mathcal{B H}}>\mathcal{S} & t_{1}<t<t_{2} \\
\mathcal{S}_{B}>\mathcal{S}_{H}>\mathcal{S}>\mathcal{S}_{\mathcal{B H}} & t_{2}<t<t_{3} \\
\mathcal{S}_{B}>\mathcal{S}>\mathcal{S}_{H}>\mathcal{S}_{\mathcal{B H}} & t>t_{3} .
\end{array}
$$


The entropy bounds $\mathcal{S}<\mathcal{S}_{B H}$ and $\mathcal{S}<\mathcal{S}_{H}$ are valid only for small $t$. On the other hand, the bound $\mathcal{S}<\mathcal{S}_{B}$ is valid only for large $t$. (Here we assume $b^{\prime}$ is negative as usually occurs even in the presence of quantum gravity.) Therefore the entropy bounds seem to break down in general due to the quantum effects. This indicates that in the region where quantum effects are dominant, one should re-consider the fundamental physical laws as it was suggested some time ago by 't Hooft.

\section{Discussion}

In summary, we were trying to clarify the role and the importance of conformal anomaly in AdS/CFT correspondence and in related issues. In particulary, the indication to the window for realization of brane quantum gravity in AdS/CFT set-up (via comparison of holographic and QFT conformal anomalies) is presented. The occurence of quantum induced inflationary (or hyperbolic) brane-world scenario in terms of AdS3/CFT2 correspondence is presented. Quantum corrected entropy for inflationary Universe is proposed and some related entropy bounds which turned out to be evolving are discussed. Again, the basic elements of this calculation are the conformal anomaly and AdS/CFT.

Of course, our study being useful in clarification of some aspects of AdS/CFT may be extended in various directions. For example, the better understanding of incorporation of Randall-Sundrum scenario to string theory is required. New Brane World realized in third section in frames of holographic flow within AdS3/CFT2 correspondence provides the useful background for such understanding. From another side, the hypotetical quantum corrected entropy bounds are estimated only for inflationary Universe (its early and late stages). It is clear that at the exit from inflationary stage these bounds should be completely modified. It would be really interesting to study their evolution especially at the end of the inflation. Moreover, it could be that even the definition of quantum entropy at early Universe should be modified. This fundamental problem deserves the very careful future investigation.

\section{Acknoweledgements}

We are very grateful to G. Gibbons, O. Obregon and V.I. Tkach for useful discussions. The work by SDO has been supported in part by CONACyT 
(CP, Ref.990356 and grant 28454E) and in part by RFBR grant N99-0216617.

\section{References}

[1] J.M. Maldacena, Adv.Theor.Math.Phys. 2 (1998) 231; E. Witten, Adv.Theor.Math.Phys. 2 (1998) 253; S. Gubser, I. Klebanov and A. Polyakov, Phys.Lett. B428 (1998) 105;

[2] M. Henningson and K. Skenderis, JHEP 9807 (1998) 023; S. Nojiri and S. D. Odintsov, Phys.Lett. B444 (1998) 92, hep-th/9810008; M. Nishimura and Y. Tanii, Int.J.Mod.Phys. A14 (1999) 3731; V. Balasubramanian and P. Kraus, Commun.Math.Phys. 208 (1999) 3731; S. Nojiri and S.D. Odintsov, Int.J.Mod.Phys. A15 (2000) 413, hep-th/9903033; Mod.Phys.Lett. A15 (2000) 1043, hep-th/9910113; M. Blau,K. Narain and E. Gava, JHEP 9909 (1999) 018; W. Muck and K.S. Wiswanathan, hep-th/9905046; P. Mansfield and D. Nolland, JHEP 9907 (1999) 028; hep-th/0005224; J. Ho, hep-th/9910124; C. Imbimbo, A. Schwimmer, S. Theisen and S. Yankielowicz, hep-th/9910267; F. Bastianelli, S. Frolov and A. Tseytlin, hep-th/0001041; S. Nojiri, S.D. Odintsov, S. Ogushi, hep-th/0001122, to appear in Phys.Rev. D; hep-th/0009015, to appear in Phys.Lett. B; M. Fukuma, S. Matsuura and T. Sakai, hep-th/0007062.

[3] M.J. Duff, Class.Quant.Grav. 11 (1994)1387.

[4] S. Nojiri, S.D. Odintsov and S. Zerbini, Phys.Rev. D62 (2000) 064006, hep-th/0001192; S. Nojiri and S.D. Odintsov, Phys.Lett. B484 (2000) 119, hep-th/0004097.

[5] S. W. Hawking, T. Hertog and H.S. Reall, Phys.Rev. D62 (2000) 043501, hep-th/0003052.

[6] A. Starobinsky, Phys.Lett. B91 (1980) 99.

[7] S. Nojiri, O. Obregon and S.D. Odintsov, hep-th/0005127, Phys.Rev. D, to appear;

[8] E. Verlinde, hep-th/0008140. 
[9] G. Gibbons, Nucl.Phys. B292 (1987) 784.

[10] L. Randall and R. Sundrum, Phys.Rev.Lett. 83 (1999) 3370, hepth/9905221; Phys.Rev.Lett. 83 (1999)4690, hep-th/9906064.

[11] I. Antoniadis, P.O. Mazur and E. Mottola, Nucl.Phys. 388 (1992) 627.

[12] S. Nojiri and S.D. Odintsov, Phys.Rev. D59 (1999) 044026, hepth/9804033.

[13] L. Anchordoqui, C. Nunez and K. Olsen, hep-th/0007064; L. Anchordoqui and K. Olsen, hep-th/0008102.

[14] S. Nojiri, S.D. Odintsov and K.E. Osetrin, hep-th/0009059.

[15] S.W. Hawking, T. Hertog and H.S. Reall, hep-th/0010232.

[16] S.W. Hawking, Comm.Math.Phys. 43 (1974) 199.

[17] G.'t Hooft, gr-qc/9312026; L. Susskind, J.Math.Phys. 36 (1995)6337.

[18] R. Bousso, JHEP 9907 (1999) 004, hep-th/9905177; JHEP 9906 (1999) 028, hep-th/9906022.

[19] S.W. Hawking, J. Maldacena and A. Strominger, hep-th/0002145.

[20] J.D. Bekenstein, Phys.Rev.D49 (1994) 1912.

[21] G. Veneziano, Phys.Lett. B454 (1999); R. Easther and D. Lowe, Phys.Rev.Lett. 82 (1999) 4967; D. Bak and S.-J. Rey, hep-th/9902173; N. Kaloper and A Linde, hep-th/9904120; R. Brushtein and G. Veneziano,Phys.Rev.Lett. 84 (2000) 5695; E. Flanagan, D. Marolf and R. Wald, hep-th/9908070;

[22] S. Nojiri and S.D. Odintsov, hep-th/0008160, PLB, to appear.

[23] D. Kutasov and F. Larsen, hep-th/0009244; F. Lin, hep-th/0010127.

[24] I.L. Buchbinder, S.D. Odintsov and I.L. Shapiro, Effective Action in Quantum Gravity, IOP Publishing, Bristol and Philadelphia, 1992.

[25] S.G. Mamaev and V.M. Mostepanenko, JETP 51 (1980) 9. 